\title{
Ueber die Transformation der Elasticitätsgleichungen in allgemeine orthogonale Coordinaten.
}

\author{
(Von C.W. Borchardt.)
}

Wir verdanken Lamé ein wichtiges Resultat in der Theorie der Elasticität fester isotroper Körper *), welches die Transformation der Differentialgleichungen für die elastischen Verrückungen in ein System allgemeiner orthogonaler Coordinaten betrift.

Dies Resultat, welches er im Liouvilleschen Journal 1841 p. 52 und in seinem berühmten Werk „Leçons sur les coordonnées curvilignes" p. 290 vermittelst einer langen, aber mit gewohnter Meisterschaft angelegten Rechnung hergeleitet hat, lässt sich in einer Form aussprechen, welche an Einfachheit nichts zu wünschen übrig lässt.

Es seien $x, y, z$ die rechtwinkligen geradlinigen Coordinaten eines Punktes eines festen elastischen Körpers im Zustande des Arsprünglichen elastischen Gleichgewichts, $x+u, y+v, z+w$ nach geschehener elastischer Deformation, dann hängt, wie man weiss, die Bestimmung von $u, v$, $w$ von drei für den ganzen elastischen Körper geltenden simultanen partiellen linearen Differentialgleichungen zweiter Ordnung und von drei für die Oberfläche des Körpers geltenden Grenzbedingungen erster Ordnung ab. Bildet man von den drei Verrückungen $u, v, w$ die neun Ableitungen nach $x, y, z$, und aus diesen die sechs Grössen

und

$$
\frac{\partial u}{\partial x}, \frac{\partial v}{\partial y}, \frac{\partial v}{\partial z}
$$

$$
\frac{\partial v}{\partial z}+\frac{\partial w}{\partial y}, \frac{\partial w}{\partial x}+\frac{\partial u}{\partial z}, \frac{\partial u}{\partial y}+\frac{\partial v}{\partial x}
$$

welche man mit Herrn Saint-Venant ${ }^{*}$ ) die drei Dilatationen und die dreiGleitungen nennen kann, so sind für elastische Körper jeder Art die Grenzbedingungen durch diese sechs Grössen selbst und die partiellen Differential-

*) d. h. deren Elasticität unabhängig von der Richtung ist.

**) Liouvilles Journal 1863, pp. 260, 262. 
gleichungen durch die nach $x, y, z$ genommenen Differentialquotienten derselben darstellbar.

Aber im Fall der Isotropie giebt es für die partiellen Differentialgleichungen eine einfachere Form. Betrachtet man ausser den sechs Dilatationen und Gleitungen die drei doppelten Componenten der ElementarRotation

$$
U=\frac{\partial v}{\partial z}-\frac{\partial w}{\partial y}, \quad V=\frac{\partial w}{\partial x}-\frac{\partial u}{\partial z}, \quad W=\frac{\partial u}{\partial y}-\frac{\partial v}{\partial x}
$$

und bildet aus den drei Dilatationen die Volumen-Dilatation

$$
p=\frac{\partial u}{\partial x}+\frac{\partial v}{\partial y}+\frac{\partial v}{\partial z},
$$

so haben die partiellen Differentialgleichungen der elastischen Verrückungen isotroper Körper die charakteristische Eigenschaft, sich aus den Differentialquotienten der vier Verbindungen $p, U, V, W$ zusammensetzen zu lassen.

Dies vorausgesetzt lässt sich das Lamésche Resultat so aussprechen, dass die auseinander gesetzte charakteristische Eigenschaft der partiellen Differentialgleichungen auch für krummlinige orthogonale Coordinaten bestehen bleibt. Bildet man für ein allgemeines orthogonales Coordinatensystem die Ausdrücke der Volumen-Dilatation und der drei im Sinne der wachsenden Coordinaten genommenen Componenten der Elementar-Rotation, so lassen sich aus diesen vier Grössen und deren Differentialquotienten nach den drei Coordinaten die partiellen Differentialgleichungen der elastischen Verrückungen isotroper Körper zusammensetzen.

Es leuchtet ein, dass ein Resultat, welches sich so einfach aussprechen lässt, ohne Aufwand von Rechnung herzuleiten sein muss.

Jacobi hat gezeigt ${ }^{*}$ ), dass die Transformation in allgemeine und namentlich in orthogonale Coordinaten für die aus der Variation eines mehrfachen Integrals hervorgehenden partiellen Differentialgleichungen erheblich vereinfacht wird, wenn man dieselbe nicht an der Differentialgleichung, sondern an dem Integral ausführt.

Diesen von Jacobi für Probleme mit einer abhängigen Variable auseinander gesetzten Gedanken hat Herr Carl Neumann auf das in der Elasticität isotroper Körper vorkommende Problem mit drei abhängigen Variablen aus-

*) Bd. 36 p. 113 dieses Journals. 
gedehnt $^{*}$ ). Aber in dem vorliegenden Falle reicht die Jacobische Methode allein nicht aus, um für die von Lamé gefundene Form der transformirten Gleichungen eine befriedigende Herleitung zu ergeben. Es bilden nämlich die Dilatationen und Gleitungen einerseits, die Elementar-Rotationen andrerseits zwei Gruppen von Grössen, für deren jede eine besondere und sehr einfache Art der Transformation besteht. Vermischt man dagegen beide Gruppen, so kann man in der Transformation solcher gemischter Grössen kein einfaches Gesetz mehr erkennen.

Auf den folgenden Seiten werde ich zeigen, dass wenn man beide Gruppen gehörig trennt und überdies bei der Transformation der Coordinaten in einander die totalen Differentiale anstatt der partiellen Differentialquotienten in Betracht zieht, das Lamésche Resultat fast ohne Rechnung erlangt wird.

1. Die Grundgleichung der Elasticität, ihre Umformung im Fall der Isotropie. Die Gleichungen der Elasticität sind von Green auf eine einzige Gleichung zurückgeführt worden, welche ausdrückt, dass die Variation eines dreifachen Integrals dem Moment der gegebenen Kräfte gleich ist. Werden die Verrückungen als unendlich klein behandelt, so heisst für den Fall isotroper Körper diese Grundgleichung in der Kirchhoffschen Bezeichnung **)

Darin bedeutet

$$
\text { (1.) } \delta P=K \delta \Omega \text {. }
$$

$\left(1^{*}.\right)$

$$
\left\{\begin{array}{c}
\delta P=\delta^{(3)} \boldsymbol{P}+\delta^{(2)} \boldsymbol{P} \\
=\int d T[X \delta u+Y \delta v+Z \delta w]+\int d \omega[(X) \delta u+(Y) \delta v+(Z) \delta w]
\end{array}\right.
$$

das Moment der gegebenen Kräfte, und zwar umfasst das über die VolumenElemente $d T$ erstreckte Integral $\delta^{(3)} P$ die auf alle inneren Punkte des Körpers wirkenden, das über die Oberflächen-Elemente $d \omega$ erstreckte Integral $\delta^{(2)} P$ die auf alle Punkte der Begrenzung des Körpers wirkenden Kräfte. $\Omega$ bedeutet das Integral

(2.)

$$
\left\{\begin{array}{l}
\Omega=\int d T\left\{\mathfrak{F}+\theta p^{2}-\mathfrak{g s p}\right\} \\
\S=\left(\frac{\partial u}{\partial x}\right)^{2}+\left(\frac{\partial v}{\partial y}\right)^{2}+\left(\frac{\partial w}{\partial z}\right)^{2}+\frac{1}{2}\left(\frac{\partial v}{\partial z}+\frac{\partial w}{\partial y}\right)^{2}+\frac{1}{2}\left(\frac{\partial w}{\partial x}+\frac{\partial u}{\partial z}\right)^{2}+\frac{1}{2}\left(\frac{\partial u}{\partial y}+\frac{\partial v}{\partial x}\right)^{2} \\
p=\frac{\partial u}{\partial x}+\frac{\partial v}{\partial y}+\frac{\partial w}{\partial z}
\end{array}\right.
$$

*) Bd. 57 p. 281 dieses Journals.

**) Bd. 40 p. 55 dieses Journals. 
$K$ und $K \boldsymbol{\theta}$ sind die beiden Constanten der Elasticität. Das in dem Integral $\Omega$ vorkommende Glied - $g s p$, welches sich in den Kirchhoffschen Untersuchungen nicht findet, ist hinzugefügt, um den von Duhamel und Herrn Franz Neumann untersuchten Fall mit zu. umfassen, in welchem eine in den einzelnen Theilen des elastischen Körpers ungleich vertheilte Erwärmung

$$
s(x, y, z)
$$

zu den elastischen Deformationen mitwirkt. Die als Factor dieses Gliedes auftretende Constante $\mathfrak{g}$ hängt durch die Gleichung

$$
\mathfrak{g}=2(1+3 \theta) \mathfrak{e}
$$

von dem linearen thermischen Ausdehnungscoefficienten $\mathfrak{e}$ des elastischen Körpers $\mathbf{a b}^{*}$ ).

Reducirt man nach den Vorschriften der Variationsrechnung die Variation $\delta \Omega$ auf ihre einfachste Form

$$
\delta \Omega=\delta^{(3)} \Omega+\delta^{(2)} \Omega,
$$

wo $\delta^{(3)}$ den aus einem Volumen-Integral, $\delta^{(2)}$ den aus einem OberflächenIntegral bestehenden Theil der Variation bezeichnet, so spaltet sich die Grundgleichung (1:) in die beiden Gleichungen

$$
\begin{array}{cc}
\left(1^{a} .\right) & \delta^{(3)} P=K \delta^{(3)} \Omega, \\
\left(1^{b} .\right) & \delta^{(2)} P=K \delta^{(2)} \Omega .
\end{array}
$$

Die letztere, welche die drei Bedingungen für die Oberfläche des elastischen Körpers in sich fasst, ist im Allgemeinen einer weiteren Vereinfachung nicht fähig. Die erstere dagegen, welche die drei partiellen Differentialgleichungen in sich fasst, lässt eine wesentliche Vereinfachung zu und zwar durch eine Umformung von $\delta^{(3)} \Omega$, welche mit der oben erwähnten im Falle der Isotropie eintretenden charakteristischen Eigenschaft der partiellen Differentialgleichungen gleichbedeutend ist.

Setzt man

$$
4 \mathfrak{F}=U^{2}+V^{2}+W^{2}, \quad \mathfrak{S}=\mathfrak{A}+\mathfrak{B}+\mathfrak{E},
$$

wo $U, V, W$ wie oben die doppelten Componenten der Elementar-Rotation

$$
\boldsymbol{U}=\frac{\partial v}{\partial z}-\frac{\partial w}{\partial y}, \quad \boldsymbol{V}=\frac{\partial w}{\partial x}-\frac{\partial u}{\partial z}, \quad W=\frac{\partial u}{\partial y}-\frac{\partial v}{\partial x}
$$

*) Man vergleiche Franz Neumann die Gesetze der Doppelbrechung des Lichts in comprimirten oder ungleichförmig erwärmten unkrystallinischen Körpern. Abhandlungen der Berliner Akademie aus dem Jahre 1841 p. 100, woselbst $\theta=\frac{1}{2}$ angenommen ist. 
und $\mathfrak{A}, \mathfrak{B}$, $\mathfrak{C}$ die Functional-Determinanten zweiter Ordnung

$$
\mathfrak{A}=\frac{\partial v}{\partial y} \frac{\partial w}{\partial z}-\frac{\partial v}{\partial z} \frac{\partial w}{\partial y}, \quad \mathfrak{B}=\frac{\partial w}{\partial z} \frac{\partial u}{\partial x}-\frac{\partial w}{\partial x} \frac{\partial u}{\partial z}, \quad \mathfrak{S}=\frac{\partial u}{\partial x} \frac{\partial v}{\partial y}-\frac{\partial u}{\partial y} \frac{\partial v}{\partial x}
$$

bedeuten, so kann man $\mathcal{F}$ unter der Form

$$
\mathbb{E}=p^{2}+2 \mathfrak{F}-2 \mathscr{S}
$$

darstellen. Führt man neben $\Omega$ die neuen Integrale

ein, so hat man

$$
\Omega^{\prime}=\int d T\left\{2 \mathfrak{F}+(1+\theta) p^{2}-g s p\right\}, \quad \Gamma=\int d T(S)
$$

$$
\Omega=\Omega^{\prime}-2 \Gamma \text {. }
$$

Das Integral $I$ ist aber kein dreifaches oder Volumen-Integral im eigentlichen Sinne, sondern ein Oberflächen-Integral. Wie nämlich ein einfaches Integral

$$
\int d x \frac{\partial f}{\partial x}
$$

unter welchem ein Differentialquotient steht, kein eigentliches Integral ist, sondern nur von den an den Grenzen der Integration stattfindenden Werthen der Function $f$ abhängt, so ist allgemein ein $n$ faches Integral

$$
\int d x_{1} \ldots d x_{n} \mathfrak{M}=\int d x_{1} \ldots d x_{n} \frac{\partial\left(f_{1} \ldots f_{m}\right)}{\partial\left(x_{1} \ldots x_{m}\right)}, \quad m \leqq n
$$

unter welchem eine nach $m$ der $n$ Integrationsvariablen $x_{1}, \ldots x_{n}$ genommene Functional-Determinante $\mathfrak{M}$ steht, kein eigentliches $n$ faches Integral, sondern höchstens ein $n-1$ faches. Denn da $\mathfrak{M}$ auf die Form

$$
\mathfrak{M}=\frac{\partial\left(f_{1} \mathfrak{M}_{1}\right)}{\partial x_{1}}+\frac{\partial\left(f_{1} \mathfrak{M}_{2}\right)}{\partial x_{2}}+\cdots+\frac{\partial\left(f_{1} \mathfrak{M}_{m}\right)}{\partial x_{m}}, \quad \mathfrak{M}_{\mu}=\frac{\partial \mathfrak{M}}{\partial \frac{\partial f_{1}}{\partial x_{\mu}}}
$$

gebracht werden kann ${ }^{*}$, so ist das betrachtete Integral nicht von den Werthen der Functionen $f_{1}, \ldots f_{m}$ innerhalb des ein $n$ faches Continuum bildenden Integrationsbereichs, sondern nur von den an den Grenzen des Continuums stattfindenden Werthen abhängig. Unter diese Categorie fällt für $n=3, m=2$ das Integral **):

$$
\Gamma=\int d x d y d z(\mathfrak{A}+\mathfrak{B}+\mathfrak{E}),
$$

*) Jacobi, theoria novi multiplicatoris Bd. 27 p. 203 dieses Journals.

**) Der Werth von $\Gamma$ als Oberflächen-Integral wird durch die Formel $2 \Gamma=\int d \omega\{[u \cdot p-\varepsilon u] \cos (\nu, x)+[v \cdot p-\varepsilon v] \cos (\nu, y)+[w \cdot p-\varepsilon v] \cos (\nu, z)\}$ gegeben, wenn man ftir irgend eine Function $f$ von $x, y$, z mit $f f$ den Ausdruck

$$
\varepsilon f=\frac{\partial f}{\partial x} u+\frac{\partial f}{\partial y} v+\frac{\partial f}{\partial z} w
$$

Journal für Mathematik Bd. LXXVI. Heft 1. 
also ist $T$ und mithin auch $\delta T$ ein blosses Oberflächen-Integral *). Mit Berücksichtigung der Gleichung

$$
\Omega=\Omega^{\prime}-2 \Gamma
$$

und wenn man die Theilung der Variationen $\delta$ in die Theile $\delta^{(3)}$ und $\delta^{(2)}$ auch für die übrigen Integrale durchführt, ergiebt sich daher $\delta^{(3)} \Gamma=0$ und

$$
\delta^{(3)} \Omega=\delta^{(3)} \Omega^{\prime} .
$$

Durch diese Umformung geht $\left(1^{a}\right.$.) in $\delta^{(3)} P=K \delta^{(3)} \Omega^{\prime}$ über. Es giebt also im Fall der Isotropie folgende vereinfachte Form, unter welcher sich die partiellen Differentialgleichungen der Elasticität in eine Gleichung zusammenfassen lassen:

$$
\left\{\begin{array}{l}
\text { (3.) } \quad \delta^{(3)} \boldsymbol{P}=K \delta^{(3)} \Omega^{\prime}, \\
\boldsymbol{4} \mathfrak{F}=\int \boldsymbol{d}=\boldsymbol{U}^{2}+V^{2}+W^{2}=\left(\frac{\partial v}{\partial z}-\frac{\partial w}{\partial y}\right)^{2}+\left(\frac{\partial w}{\partial x}-\frac{\partial u}{\partial z}\right)^{2}+\left(\frac{\partial u}{\partial y}-\frac{\partial v}{\partial x}\right)^{2} \\
p=\frac{\partial u}{\partial x}+\frac{\partial v}{\partial y}+\frac{\partial w}{\partial z} .
\end{array}\right.
$$

2. Transformation der linearen Dilatationen. Im Folgenden werde ich, wie bereits in $\$$. 1, den Buchstaben $\varepsilon$ als Operationszeichen für die elastischen Variationen brauchen, sodass, wenn $f$ irgend eine Function der Coordinaten bedeutet, $f+\varepsilon f$ den Werth von $f$ nach geschehener elastischer Deformation bedeuten soll.

Die in unendlich kleiner Entfernung vom Punkte $(x, y, z)$ stattfindenden Verrückungen kann man bekanntlich aus zwei Arten von Veränderungen des Elementes $d T$ zusammensetzen, von welchen die erste Art in den linearen Dilatationen ohne Drehung besteht.

Es seien $x^{\prime}, y^{\prime}, z^{\prime}$ die Coordinaten eines zum Element $d T$ gehörigen Punktes in seiner ursprünglichen Lage, $r$ die Länge der von $(x, y, z)$ nach $\left(x^{\prime}, y^{\prime}, z^{\prime}\right)$ gezogenen Geraden, $\alpha, \alpha_{1}, \alpha_{2}$ die Cosinus der Winkel, welche diese Gerade mit den Richtungen der wachsenden $x, y, z$ bildet. Bei der elastischen Deformation verwandle sich $r$ in $r+\varepsilon r$, so ist bekanntlich die lineare

und mit $(\nu, x),(\nu, y),(\nu, z)$ die Winkel bezeichnet, welche die nach Aussen gerichtete Normale des Oberflächen-Elements $d \omega$ mit den positiven Seiten der Coordinatenaxen bildet.

*) Der besondere Fall, in welchem sich das Integrationsgebiet von $\boldsymbol{\Gamma}$ noch weiter einschränkt, kommt hier nicht in Betracht. 
Dilatation $\frac{\varepsilon r}{r}$ durch die Gleichung

$$
\frac{\varepsilon r}{r}=\Sigma a_{i k} \alpha_{i} \alpha_{k} \quad(i, k=0,1,2)
$$

gegeben, wo

und

$$
\alpha^{2}+\alpha_{1}^{2}+\alpha_{2}^{2}=1
$$

$$
\begin{array}{lll}
\boldsymbol{a}_{(1)}=\frac{\partial u}{\partial x}, & \boldsymbol{a}_{11}=\frac{\partial v}{\partial y}, & \boldsymbol{a}_{22}=\frac{\partial v}{\partial z}, \\
\boldsymbol{a}_{12}=\frac{1}{2}\left(\frac{\partial v}{\partial z}+\frac{\partial w}{\partial y}\right), & \boldsymbol{a}_{112}=\frac{1}{2}\left(\frac{\partial v}{\partial x}+\frac{\partial u}{\partial z}\right), & \boldsymbol{a}_{01}=\frac{1}{2}\left(\frac{\partial u}{\partial y}+\frac{\partial v}{\partial x}\right) .
\end{array}
$$

Die beiden in dem Integral $\Omega$ vorkommenden Ausdrücke $\leftleftarrows, p$ lassen sich in den Coefficienten $a_{i k}$ in der Form *)

$$
\begin{gathered}
\xi=\sum_{i k} a_{i k}^{2}, \\
p=\sum_{i} a_{i i}
\end{gathered}
$$

darstellen, sie sind simultane Invarianten der beiden quadratischen Formen

$$
\sum_{i k} a_{i k} \alpha_{i} \alpha_{k}, \quad \sum_{i} \alpha_{i}^{2}
$$

und bleiben daher für alle orthogonalen Transformationen unverändert, $d$. h. stellt man die lineare Dilatation in irgend einem gerad- oder krummlinigen orthogonalen Coordinatensystem unter der Form

$$
\frac{\varepsilon r}{r}=\sum_{i k} b_{i k} \beta_{i} \beta_{k}
$$

dar, wo $\beta, \beta_{1}, \beta_{2}$ die diesem Coordinatensystem entsprechenden Richtungscosinus bedeuten, so haben $₹$ und $p$ in den Grössen $b_{i k}$ dieselben Ausdrücke, wie in den Grössen $a_{i k}$.

Es seien $\varrho, \varrho_{1}, \varrho_{2}$ drei Functionen von $x, y, z$, welche ein orthogonales Coordinatensystem bilden, also $d \rho d \varrho_{1}, d \varrho_{2}$ lineare Functionen von $d x, d y, d z$, welche der Gleichung

genügen.

$$
d x^{2}+d y^{2}+d z^{2}=\frac{d \varrho^{2}}{h^{2}}+\frac{d \varrho_{1}^{2}}{h_{1}^{2}}+\frac{d \varrho_{2}^{2}}{h_{2}^{2}}
$$

In dem geradlinigen rechtwinkligen Coordinatensystem der $x, y, z$ sind $u, v, w$ zugleich die elastischen Variationen der Coordinaten und die

*) In den im Folgenden vorkommenden einfachen oder Doppel-Summen sind dem Index $i$ oder $k$ oder beiden die Werthe $0,1,2$ beizulegen. 
Verrückungen im Sinne derselben. Im System der $\varrho, \varrho_{1}, \varrho_{2}$ ist dies nicht mehr der Fall, hier stehen die elastischen Variationen $\varepsilon \varrho_{1} \varepsilon \varrho_{1}, \varepsilon \varrho_{2}$ der Coordinaten und die Verrückungen $R, R_{1}, R_{2}$ im Sinne der wachsenden $\varrho, \rho_{1}, \rho_{2}$ durch die Gleichungen

$$
\boldsymbol{R}_{i}=\frac{\varepsilon \varrho_{i}}{h_{i}}
$$

in Verbindung mit einander.

Die Coordinaten der beiden unendlich nahen Punkte $(x, y, z)$ und $\left(x^{\prime}, y^{\prime}, z^{\prime}\right)$ im neuen Systeme bezeichne ich mit $\varrho, \varrho_{1}, \varrho_{2}$ und $\varrho^{\prime}, \varrho_{1}^{\prime}, \varrho_{2}^{\prime}$; dann ist ihre Entfernung $r$ durch die Gleichung

$$
r^{2}=\left(\frac{\varrho^{\prime}-\varrho}{h}\right)^{2}+\left(\frac{\varrho_{1}^{\prime}-\varrho_{1}}{h_{1}}\right)^{2}+\left(\frac{\varrho_{2}^{\prime}-\varrho_{2}}{h_{2}}\right)^{2}
$$

gegeben. Nach geschehener elastischer Deformation geht $r^{2}$ in

$$
(r+\varepsilon r)^{2}=\left(\frac{\varrho^{\prime}-\varrho+\varepsilon \varrho^{\prime}-\varepsilon \varrho}{h+\varepsilon h}\right)^{2}+\left(\frac{\varrho_{1}^{\prime}-\varrho_{1}+\varepsilon \varrho_{1}^{\prime}-\varepsilon \varrho_{1}}{h_{1}+\varepsilon h_{1}}\right)^{2}+\left(\frac{\varrho_{2}^{\prime}-\varrho_{2}+\varepsilon \varrho_{2}^{\prime}-\varepsilon \varrho_{2}}{h_{2}+\varepsilon h_{2}}\right)^{2}
$$

über. Von den drei Brüchen, deren Quadrate die rechte Seite dieser Gleichung bilden, transformirt man den ersten, da die elastischen Variationen als unendlich klein $\mathrm{zu}$ behandeln sind, vermöge der Gleichungen

in

$$
\begin{aligned}
& \frac{\varrho^{\prime}-\varrho+\varepsilon \varrho^{\prime}-\varepsilon \varrho}{h+\varepsilon h}=\frac{1}{h}\left\{\left(1-\frac{\varepsilon h}{h}\right)\left(\varrho^{\prime}-\varrho\right)+\varepsilon \varrho^{\prime}-\varepsilon \rho\right\}, \\
& \varepsilon \rho^{\prime}-\varepsilon \varphi=\frac{\partial \varepsilon \rho}{\partial \rho}\left(\rho^{\prime}-\rho\right)+\frac{\partial \varepsilon \rho}{\partial \varrho_{1}}\left(\varrho_{1}^{\prime}-\varrho_{1}\right)+\frac{\partial \varepsilon \rho}{\partial \rho_{2}}\left(\rho_{2}^{\prime}-\rho_{2}\right)
\end{aligned}
$$

$$
\frac{\varrho^{\prime}-\varrho+\varepsilon \varrho^{\prime}-\varepsilon \varrho}{h+\varepsilon h}=\left(1+\frac{\partial \varepsilon \varrho}{\partial \varrho}-\frac{\varepsilon h}{h}\right) \frac{\varrho^{\prime}-\varrho}{h}+\frac{\partial \varepsilon \varrho}{\partial \varrho_{1}} \frac{\varrho_{1}^{\prime}-\varrho_{1}}{h}+\frac{\partial \varepsilon \varrho}{\partial \varrho_{2}} \frac{\varrho_{2}^{\prime}-\varrho_{2}}{h} .
$$

Indem man diesen Ausdruck und die beiden ähnlich gebildeten in die obige Gleichung für das Quadrat von $r+\varepsilon r$ einsetzt, ergiebt sich für die Dilatation $\frac{\varepsilon r}{r}$ das Resultat

$$
\frac{\imath r}{r}=\sum_{i k} b_{i k} \beta_{i} \beta_{k}
$$

wo

$$
\begin{array}{ll}
b_{i i}=\frac{\partial \varepsilon \varrho_{i}}{\partial \varrho_{i}}-\frac{\varepsilon h_{i}}{h_{i}} ; & b_{i k}=\frac{i}{2}\left(\frac{h_{i}}{h_{k}} \frac{\partial \varepsilon \varrho_{k}}{\partial \varrho_{i}}+\frac{h_{k}}{h_{i}} \frac{\partial \varepsilon \varrho_{i}}{\partial \varrho_{k}}\right), \\
\beta_{i}=\frac{\varrho_{i}^{\prime}-\rho_{i}}{r h_{i}}, \quad & \beta^{2}+\beta_{1}^{2}+\beta_{2}^{2}=1 .
\end{array}
$$


Da $\mathcal{E}$ und $p$ dieselben Ausdrücke in $b_{i k}$ wie in $a_{i k}$ haben, so ist

$$
\xi=\sum_{i k} b_{i k}^{2}, \quad p=\sum_{i} b_{i i} \text {. }
$$

Hiermit sind die im Integral $\Omega$ vorkommenden Grössen in das. Coordinatensystem der $\varrho, \varrho_{1}, \varrho_{2}$ transformirt, und der neue Ausdruck dieses Integrals wird, wenn man zur Abkürzung

setzt, der folgende:

$$
\mathfrak{r}_{i}=\varepsilon \varrho_{i}
$$

$$
\text { (5.) } \quad\left\{\begin{array}{l}
\Omega=\int d T\left\{\xi+\theta p^{2}-\mathfrak{g} s p\right\}, \quad d T=\frac{d \varrho d \varrho_{1}}{\varpi} \frac{d \varrho_{2}}{\varpi}, \quad \widetilde{\omega}=h h_{1} h_{2}, \\
\varsubsetneqq=\sum_{i k} b_{i k}^{2}, \quad b_{i i}=\frac{\partial \mathfrak{r}_{i}}{\partial \varrho_{i}}-\frac{1}{h_{i}} \sum_{k} \frac{\partial h_{i}}{\partial \varrho_{k}} \mathfrak{r}_{k}, \quad b_{i k}=\frac{1}{2}\left(\frac{h_{i}}{h_{k}} \frac{\partial \mathfrak{r}_{k}}{\partial \varrho_{i}}+\frac{h_{k}}{h_{i}} \frac{\partial \mathfrak{r}_{i}}{\partial \varrho_{k}}\right), \\
p=\sum_{i} b_{i i}=\widetilde{\omega} \sum_{i} \frac{\partial}{\partial \varrho_{i}}\left(\frac{\mathfrak{r}_{i}}{\varpi}\right), \quad \mathfrak{r}_{i}=h_{i} R_{i} .
\end{array}\right.
$$

3. Transformation der Elementar-Rotationen. In diesem Paragraphen soll die durch die elastischen Deformationen bewirkte zweite Art von Veränderungen des Volumen-Elementes $d T$ betrachtet werden, welche in einer Drehung des Elementes ohne lineare Dilatationen besteht. Die doppelten Werthe der drei Componenten dieser Drehung um die Axen der $x, y, z$ sind

$$
U=\frac{\partial v}{\partial z}-\frac{\partial w}{\partial y}, \quad V=\frac{\partial w}{\partial x}-\frac{\partial u}{\partial z}, \quad W=\frac{\partial u}{\partial y}-\frac{\partial v}{\partial x},
$$

daher ist das Quadrat der ganzen Drehung identisch mit der im Integral $\Omega^{\prime}$ vorkommenden Grösse $\mathfrak{F}$, welche durch die Gleichung

$$
4 \mathfrak{F}=U^{2}+V^{2}+W^{2}
$$

definirt wurde. Diese physikalische Bedeutung lässt schon von vorn herein in $\mathfrak{F}$ eine Invariante bei der Transformation in allgemeine orthogonale Coordinaten erkennen, aber der invariantive Charakter von $\mathfrak{F}$ ist verschieden von dem der oben transformirten Grössen $\mathfrak{F}, p$. Die weiteren Entwickelungen werden zeigen, dass die beiden Arten der Invariabilität in einem adjungirten Verhältniss zu einander stehen.

Betrachtet man neben den Differentialen der Coordinaten irgend eine andere Art unendlich kleiner Aenderungen, welche die beiden Grössensysteme $x, y, z$ und $\varrho_{,} \varrho_{1}, \varrho_{2}$ gleichzeitig erleiden, und bezeichnet diese Aenderungen mit $\delta x, \delta y, \delta z$ und $\delta \varrho_{1} \delta \varrho_{1}, \delta \varrho_{2}$, so stehen diese Variationen in denselben linearen Beziehungen zu einander, wie die Differentiale, sie erfüllen daher 
auch die nämliche Bedingung zweiten Grades, und man hat gleichzeitig

$$
\begin{aligned}
& d x^{2}+d y^{2}+d z^{2}=\frac{d \rho^{2}}{h^{2}}+\frac{d \rho_{1}^{2}}{h_{1}^{2}}+\frac{d \rho_{2}^{2}}{h_{2}^{2}}, \\
& \delta x^{2}+\delta y^{2}+\delta z^{2}=\frac{\delta \rho^{2}}{h^{2}}+\frac{\delta \rho_{1}^{2}}{h_{1}^{2}}+\frac{\delta \rho_{2}^{2}}{h_{2}^{2}} .
\end{aligned}
$$

Aus der Uebereinstimmung der linearen Beziehungen folgt überdies, wie man sich leicht überzeugt, die dritte Gleichung

$$
d x \delta x+d y \delta y+d z \delta z=\frac{d \varrho \delta Q}{h^{2}}+\frac{d \varrho_{1} \delta \varrho_{1}}{h_{1}^{2}}+\frac{d \varrho_{2} \delta \varrho_{2}}{h_{1}^{2}} .
$$

Multiplicirt man die beiden ersten dieser drei Gleichungen unter Anwendung der bekannten Formel für die Darstellung des Products zweier Summen dreier Quadrate als Summe von vier Quadraten und zieht von dem Resultat die quadrirte dritte Gleichung ab, so ergiebt sich

$$
\mathfrak{X}^{2}+\mathfrak{Y}^{2}+\mathfrak{Z}^{2}=\mathfrak{R}^{2}+\mathfrak{R}_{1}^{2}+\Re_{2}^{2},
$$

wo

$$
\begin{aligned}
& \mathfrak{X}=d y \delta z-d z \delta y, \quad \mathfrak{Y}=d z \delta x-d x \delta z, \quad 3=d x \delta y-d y \delta x, \\
& \Re=\frac{d \varrho_{1} \delta \varrho_{2}-d \varrho_{2} \delta \varrho_{1}}{h_{1} h_{2}}, \quad \Re_{1}=\frac{d \varrho_{2} \delta \varrho-d \varrho \delta \varrho_{2}}{h h_{2}}, \quad \Re_{2}=\frac{d \varrho \delta \varrho_{1}-d \varrho_{1} \delta \varrho}{h h_{1}} .
\end{aligned}
$$

Indem man in

$$
d x \delta x+d y \delta y+d z \delta z=\frac{d \varrho \delta \varrho}{h^{2}}+\frac{d \varrho_{1} \delta \varrho_{1}}{h_{1}^{2}}+\frac{d \varrho_{2} \delta \varrho_{2}}{h_{2}^{2}}
$$

die Variationen $\delta$ in elastische Variationen $\varepsilon$ übergehen lässt und

setzt, erhält man

$$
\sigma_{i}=\frac{\varepsilon \rho_{i}}{h_{i}^{2}}=\frac{R_{i}}{h_{i}}
$$

$$
u d x+v d y+v d z=\sigma d \rho+\sigma_{1} d \rho_{1}+\sigma_{2} d \rho_{2}
$$

oder, wenn man zur Abkürzung

$$
f(d x)=u d x+v d y+w d z, \quad g(d \varrho)=\sigma d \rho+\sigma_{1} d \rho_{1}+\sigma_{2} d \varrho_{2}
$$

setzt,

$$
f(d x)=g(d \varrho)
$$

Diese Gleichung wird durch die linearen Beziehungen zwischen $d x, d y, d z$ und $d \rho_{,} d \rho_{1}, d \varrho_{2}$ identisch befriedigt, sie besteht daher auch, wenn für die Differentiale Variationen gesetzt werden, man hat also

$$
f(\delta x)=g(\delta \varrho)
$$

Indem man die erste dieser Gleichungen variirt, die zweite differentiirt und 
aus beiden Resultaten die Differenz bildet, ergiebt sich *)

$$
\delta f(d x)-d f(\delta x)=\delta g(d \varrho)-d g(\delta \varrho)
$$

oder in entwickelter Form

$$
\mathfrak{X} U+\mathfrak{Y} V+\mathfrak{g} W=\mathfrak{R} \mathfrak{S}+\Re_{1} \mathfrak{S}_{1}+\Re_{2} \mathfrak{S}_{2},
$$

wo

$$
\mathfrak{S}=h_{1} h_{2}\left(\frac{\partial \sigma_{1}}{\partial \varrho_{2}}-\frac{\partial \sigma_{2}}{\partial \varrho_{1}}\right), \quad \mathfrak{S}_{1}=h h_{2}\left(\frac{\partial \sigma_{2}}{\partial \varrho}-\frac{\partial \sigma}{\partial \varrho_{2}}\right), \quad \mathfrak{S}_{2}=h h_{1}\left(\frac{\partial \sigma}{\partial \varrho_{1}}-\frac{\partial \sigma_{1}}{\partial \varrho}\right) .
$$

Aber man hat folgenden bekannten algebraischen Satz:

Sind $\mathfrak{X}, \mathfrak{Y}, \mathfrak{Z}$ und $\Re, \Re_{1}, \Re_{2}$ zwei Systeme von Variablen, welche linear von einander abhängen und zugleich der Bedingung

$$
\mathfrak{X}^{2}+\mathfrak{Y}^{2}+\mathfrak{Z}^{2}=\mathfrak{R}^{2}+\mathfrak{R}_{1}^{2}+\mathfrak{R}_{2}^{2}
$$

genügen, und stehen diese beiden Systeme mit zwei anderen Systemen $U, V$, $W$ und $\mathfrak{S}_{,} \mathfrak{S}_{1}, \mathfrak{S}_{2}$ durch die identische Gleichung

$$
\mathfrak{X}+\mathfrak{Y} V+3 W=\Re \mathfrak{S}+\Re_{1} \mathfrak{S}_{1}+\Re_{2} \mathfrak{S}_{2}
$$

in Verbindung, so sind die neuen Systeme ebenfalls linear von einander abhängig und genügen ebenfalls der Bedingung

$$
U^{2}+V^{2}+W^{2}=\mathfrak{S}^{2}+\mathfrak{S}_{1}^{2}+\mathfrak{S}_{2}^{2}
$$

Hiermit ist die Transformation von

$$
2 \mathfrak{F}=\frac{1}{2}\left(U^{2}+V^{2}+W^{2}\right)=\frac{1}{2}\left(\mathfrak{S}^{2}+\mathfrak{S}_{1}^{2}+\mathfrak{S}_{2}^{2}\right)
$$

in die neuen Coordinaten ausgeführt, die Grössen $\frac{1}{2} \mathfrak{S}_{,} \frac{1}{2} \mathfrak{S}_{1}, \frac{1}{2} \mathfrak{S}_{2}$ sind, wie man sich leicht überzeugt, die Componenten der Elementar-Rotation um die Richtungen der wachsenden $\varrho, \varrho_{1}, \varrho_{2}$. Da $p$ bereits in $\$ .2$ transformirt worden ist, so sind jetzt alle in das Integral $\Omega^{\prime}$ eintretenden Grössen in die neuen Coordinaten ausgedrückt, und es ergiebt sich für $\Omega^{\prime}$ der Werth

(6.)

$$
\left\{\begin{array}{l}
\Omega^{\prime}=\int d T\left\{2 \mathfrak{F}+(1+\theta) p^{2}-\mathfrak{g} s p\right\}, \quad d T=\frac{d \varrho d \varrho_{1} d \varrho_{2}}{\varpi}, \quad \widetilde{\omega}=h h_{1} h_{2}, \\
4 \mathfrak{F}=\sum_{i} \mathfrak{S}_{i}^{2}, \quad \mathfrak{S}_{i}=h_{k} h_{l}\left(\frac{\partial \sigma_{k}}{\partial \varrho_{l}}-\frac{\partial \sigma_{l}}{\partial \varrho_{k}}\right), \\
p=\widetilde{\omega} \sum_{i} \frac{\partial}{\partial \varrho_{i}}\left(\frac{h_{i}^{2} \sigma_{i}}{\varpi}\right), \quad \sigma_{i}=\frac{R_{i}}{h_{i}},
\end{array}\right.
$$

wo $i k l$ eine positive Permutation der Indices 012 bedeutet.

Der Transformation für die Verrückungen, welche in der oben ge-

*) Man vergleiche die Abhandlung des Herrn Lipschitz, Bd. 70, p. 77 dieses Journals. 
brauchten Gleichung

$$
u d x+v d y+w d z=\sum_{i} \sigma_{i} d \varrho_{i}=\sum_{i} \frac{R_{i}}{h_{i}} d \varrho_{i}
$$

enthalten ist, lässt sich eine ähnliche für die Momente der gegebenen Kräfte an die Seite stellen, nämlich

$$
X \delta u+Y \delta v+Z \delta w=\sum_{i} \frac{P_{i}}{h_{i}} \delta r_{i}=\sum_{i} P_{i} h_{i} \delta \sigma_{i},
$$

und man transformirt demgemäss die Momente der gegebenen Kräfte durch die Gleichungen

$$
\left\{\begin{array}{l}
\delta^{(2)} P=\int d w[(X) \delta u+(Y) \delta v+(Z) \delta w]=\int d w \sum_{i} \frac{\left(P_{i}\right)}{h_{i}} \delta \mathfrak{r}_{i}, \\
\delta^{(3)} P=\int d T[X \delta u+Y \delta v+Z \delta w]=\int d T \sum_{i} P_{i} h_{i} \delta \sigma_{i} .
\end{array}\right.
$$

wo $P_{i}$ und $\left(P_{i}\right)^{\prime}$ die Componenten der gegebenen inneren und äusseren Kräfte im Sinne der wachsenden $\varrho_{i}$ bedeuten.

4. Die partiellen Differentialgleichungen und Bedingungen für die Oberfläche in allgemeinen orthogonalen Coordinaten. Um die partiellen Differentialgleichungen und Bedingungen für die Oberfläche in fertiger Form zu erhalten, kommt es jetzt noch auf die Entwickelung der Variationen $\delta^{(2)} \Omega$ und $\delta^{(3)} \Omega^{\prime}$ an. Ist ein Integral

$$
\Omega=\int d T f\left(\cdots \varrho_{k} \cdots r_{i} \cdots \frac{\partial \mathfrak{r}_{i}}{\partial \varrho_{k}} \cdots\right), \quad d T=\frac{d \varrho d \varrho_{1} d \varrho_{2}}{\sigma} \quad(i, k=0,1,2)
$$

vorgelegt, sọ ist bekanntlich die schliessliche Gestalt seiner Variation

$$
\delta \Omega=\sum_{i} \int d \boldsymbol{T} \delta \mathfrak{r}_{i}\left[f^{i}-\sum_{k} \tilde{\omega} \frac{\partial}{\partial \varrho_{k}}\left(\frac{1}{\sigma} f_{k}^{i}\right)\right]+\sum_{i} \int d \omega \delta \mathfrak{r}_{i} \sum_{k} \frac{1}{h_{k}} f_{k}^{i} \cos \left(\nu, \varrho_{k}\right),
$$

wo

$$
f^{i}=\frac{\partial f}{\partial \mathfrak{r}_{i}}, \quad f_{k}^{i}=\frac{\partial f}{\partial \frac{\partial \mathfrak{r}_{i}}{\partial \rho_{k}}}
$$

und $\left(\nu, \varrho_{k}\right)$ den Winkel bedeutet, welchen die nach Aussen gerichtete Normale des Oberflächen-Elements $d \omega$ mit der Richtung der wachsenden $\varrho_{k}$ bildet. Daher hat man

$$
\delta^{(2)} \Omega=\sum_{i}^{\Sigma} \int d \omega \delta \mathfrak{r}_{i} \underset{k}{\Sigma} \frac{1}{h_{k}} f_{k}^{i} \cos \left(\nu, \varrho_{k}\right)
$$

wo

Ebenso hat man

$$
f=\Subset+\theta p^{2}-g s p
$$




$$
\begin{gathered}
-\delta^{(3)} \Omega^{\prime}=\sum_{i} \int_{d T} \delta \sigma_{i}\left[-F^{i}+\sum_{k} \widetilde{\varpi} \frac{\partial}{\partial \varrho_{k}}\left(\frac{1}{\varpi} F_{k}^{i}\right)\right], \\
F=2 \mathfrak{F}+(1+\theta) p^{2}-g s p, \\
F^{i}=\frac{\partial F}{\partial \sigma_{i}}, \quad F_{k}^{i}=\frac{\partial F}{\partial \frac{\partial \sigma_{i}}{\partial \varrho_{k}}} .
\end{gathered}
$$

Setzt man in $f$ für $\Subset$ und $p$ ihre Ausdrücke

$$
\begin{gathered}
£=\sum_{i k} b_{i k}^{2}, \quad b_{i i}=\frac{\partial \mathfrak{r}_{i}}{\partial \varrho_{i}}-\frac{1}{h_{i}} \sum_{k} \frac{\partial h_{i}}{\partial \varrho_{k}} \mathfrak{r}_{k}, \quad b_{i k}=\frac{1}{2}\left[\frac{h_{i}}{h_{k}} \frac{\partial \mathfrak{r}_{k}}{\partial \varrho_{i}}+\frac{h_{k}}{h_{i}} \frac{\partial \mathfrak{r}_{i}}{\partial \varrho_{k}}\right], \\
p=\sum_{i} b_{i i}=\widetilde{\omega} \sum_{i} \frac{\partial}{\partial \varrho_{i}}\left(\frac{\mathfrak{r}_{i}}{\varpi}\right)
\end{gathered}
$$

ein, so ergeben sich für die in $\delta^{(2)} \Omega$ vorkommenden Ableitungen $f_{i}^{i}, f_{k}^{i}$ von $f$ die Werthe

$$
f_{i}^{i}=2\left[b_{i i}+\theta p-\frac{1}{2} g s\right], \quad f_{k}^{i}=2 b_{i k} \frac{h_{k}}{h_{i}}
$$

und daher

$$
\delta^{(2)} \Omega=2 \sum_{i} \int d \omega \frac{1}{h_{i}} \sum_{k} c_{i k} \cos \left(\nu, \varrho_{k}\right)
$$

wo

$$
c_{i i}=b_{i i}+\theta p-\frac{1}{2} g s, \quad c_{i k}=b_{i k} .
$$

Substituirt man diesen Werth von $\delta^{(2)} \Omega$ und den Werth (7.) von $\delta^{(2)} P$ in die Grundgleichung

$$
\delta^{(2)} P=K \delta^{(2)} \Omega
$$

für die Bedingungen an der Oberfläche, so erhält man die drei Bedingungsgleichungen für die Oberfläche, in den neuen Coordinaten ausgedrückt, in der sie alle drei darstellenden Form:

$$
\left[b_{i i}+\theta p-\frac{1}{2} g s\right] \cos \left(\nu, \varrho_{i}\right)+b_{i k} \cos \left(\nu, \varrho_{k}\right)+b_{i l} \cos \left(\nu, \varrho_{l}\right)=\frac{1}{2 K}\left(\mathbf{P}_{i}\right),
$$

wo $i k l$ eine Permutation der Indices 012 bedeutet und

$$
\left(8^{*} .\right) \quad b_{i i}=\frac{\partial \mathfrak{r}_{i}}{\partial \varrho_{i}}-\frac{1}{h_{i}} \sum_{k} \frac{\partial h_{i}}{\partial \varrho_{i k}} \mathfrak{r}_{k}, \quad b_{i k}=\frac{1}{2}\left[\frac{h_{i}}{h_{k}} \frac{\partial \mathfrak{r}_{k}}{\partial \varrho_{i}}+\frac{h_{k}}{h_{i}} \frac{\partial \mathfrak{r}_{i}}{\partial \varrho_{k}}\right],
$$

ein Resultat, welches, wenn $s=0$ gesetzt wird, mit den Laméschen Formeln p. 281, 282 seiner Leçons sur les coordonnées curvilignes übereinstimmt.

Setzt man in $F$ für $\mathfrak{F}$ und $p$ ihre Ausdrücke

$$
\mathbf{4} \mathfrak{F}=\sum_{i} \mathfrak{S}_{i}^{2}, \quad \mathfrak{S}_{i}=h_{k} h_{l}\left(\frac{\partial \sigma_{k}}{\partial \varrho_{l}}-\frac{\partial \sigma_{l}}{\partial \varrho_{k}}\right), \quad p=\widetilde{\omega} \sum_{i}\left(\frac{h_{i}^{2}}{\varpi} \frac{\partial \sigma_{i}}{\partial \varrho_{i}}+\sigma_{i} \frac{\partial}{\partial \varrho_{i}} \frac{h_{i}^{2}}{\varpi}\right),
$$

Journal für Mathematik Bd. LXXVI. Heft 1. 
wo $i k l$ eine positive Permutation von 012 bedeutet, und führt die Grösse

$$
q=2(1+\theta) p-g s
$$

ein, so ergeben sich für die in $\delta^{(3)} \Omega^{\prime}$ vorkommenden Ableitungen $F^{i}, F_{i}^{i}, F_{k}^{i}$ von $F$ die Werthe

$$
F^{i}=\widetilde{\varpi} \frac{\partial}{\partial \varrho_{i}} \frac{h_{i}^{2}}{\varpi} \cdot q, \quad F_{i}^{i}=h_{i}^{2} q, \quad F_{k}^{i}=(i k l) h_{i} h_{k} \mathfrak{S}_{l}^{\prime},
$$

wo $(i k l)$ die positive oder negative Einheit bedeutet, je nachdem $i k l$ eine positive oder negative Permutation von 012 ist. Demnach erhält man für $\delta^{(3)} \Omega^{\prime}$ den schliesslichen Ausdruck:

$$
-\delta^{(3)} \Omega^{\prime}=\sum_{i} \int d T \delta \sigma_{i}\left\{h_{i}^{2} \frac{\partial q}{\partial \varrho_{i}}+(i k l) \widetilde{\omega}\left[\frac{\partial}{\partial \varrho_{k}} \frac{S_{l}}{h_{l}}-\frac{\partial}{\partial \varrho_{l}} \frac{\mathfrak{S}_{k}}{h_{k}}\right]\right\} .
$$

Substituirt man diesen Werth von $\delta^{(3)} \Omega^{\prime}$ und den Werth (7.) von $\delta^{(3)} P$ in die Grundgleichung

$$
\delta^{(3)} P=K \delta^{(3)} \Omega^{\prime}
$$

für die partiellen Differentialgleichungen, und setzt fest, dass $i k l$ eine positive Permutation von 012 sein soll, so ergeben sich die drei partiellen Differentialgleichungen, in den neuen Coordinaten ausgedrückt, in der sie alle drei darstellenden Form:

$$
\text { (9.) } \quad \frac{\partial}{\partial \varrho_{l}} \frac{\mathfrak{S}_{k}}{h_{k}}-\frac{\partial}{\partial \varrho_{k}} \frac{\mathscr{S}_{l}}{h_{l}}=\frac{h_{i}}{h_{k} h_{l}} \frac{\partial q}{\partial \varrho_{i}}+\frac{1}{K} \cdot \frac{P_{i}}{h_{k} h_{l}},
$$

wo $i k l$ eine positive Permutation von 012 bedeutet, und

$$
\left(9^{*} .\right) \quad \mathfrak{S}_{i}=h_{k} h_{l}\left(\frac{\partial \sigma_{k}}{\partial \varrho_{l}}-\frac{\partial \sigma_{l}}{\partial \varrho_{k}}\right), q=2(1+\theta) p-g s, p=\widetilde{\omega} \sum_{i} \frac{\partial}{\partial \rho_{i}}\left(\frac{h_{i}^{2} \sigma_{i}}{\sigma}\right), \sigma_{i}=\frac{R_{i}}{h_{i}} .
$$

Diese Gleichungen stimmen, wenn $s=\mathbf{0}$ gesetzt wird, mit den Laméschen Systemen (25.), (26.), (27.), p. 290, 291 seiner Leçons sur les coordonnées curvilignes überein.

Berlin, im Januar 1873. 